\title{
DISTANCE PROTECTION SCHEME FOR TRANSMISSION LINE USING BACK-PROPAGATION NEURAL NETWORK
}

\author{
Himani Mahajan", Ashish Sharma ${ }^{2}$ \\ ${ }^{1}$ Department of Electrical Engineering, Lovely Professional University, Punjab, India \\ ${ }^{2}$ Department of Electrical Engineering, Lovely Professional University, Punjab, India
}

\begin{abstract}
To maintain the continuity of service to consumers without any interruption, a well coordinated protective scheme is required. A corrective decision should be made by the protective device to minimize the period of trouble and limit outage time, damage and related problems.

In this paper, back propagation feed forward neural network is considered for implementing distance protection scheme. Results obtained shows that this scheme is able to distinguish the condition and type of fault and also locates the zone in which fault is present in order to isolate the fault. All types of faults are discussed i.e. single phase to ground faults, double phase faults and double phase to ground faults. The result provides a reliable and an attractive approach for the development of a protective relaying system for the transmission system. Simulation is done using sim power system and neural network in MATLAB.
\end{abstract}

Keywords-ANN, back-propagation, transmission line, protection and fault.

****.

\section{INTRODUCTION}

Among various power system components, transmission line is one of the most important component of the power system network and is mostly affected by various types of faults. Generally, 80-90 \% of the fault occurs on the transmission line and rest on the substation equipments and bus bar combined. The necessary requirement of all the power system is to maintain reliability of operation which may be done by detecting, classifying and isolating various faults occurring in the system. It is required that a corrective decision should be made by the protective device to minimize the period of trouble and limit outage time, damage and related problems. And, if any fault or disturbances occurred in the transmission lines \& not detected, located \& eliminated quickly, it may cause instability in the power system and causes significant changes in the system quantities like over-current, under or over voltage, power factor, impedance, frequency and power. The appropriate percentage of occurrence of single line to ground fault is about $70-80 \%$, line-to-line to ground fault is $10-17 \%$, line-to- line fault is $8-10 \%$ and three phase fault is $3 \%$. The three phase fault occurs rarely but if it exists in a system it is quite dangerous.

Thus, a well coordinated protective scheme is required to maintain the continuity of service to consumers without any interruption. ANN based distance relay is considered in this paper. The operation of distance protection is based on the fact that whenever fault is occurred, the distance between any point in the power system and the fault is proportional to the ratio of voltage to current at that point.
Artificial neural network is inspired by biological neural network and is composed of number of interconnected units known as artificial neurons. Artificial neurons are used to transmit signal from one layer to other. ANN consists of three layers i.e. input layer, hidden layer and output layer having number of neurons present in it. The various advantages of ANN make it suitable to apply it to such applications. ANN performs task faster than other conventional techniques as the neurons operate at the same time leading parallel structure. ANN can dynamically adapt to changing system configurations and doesn't require any communication link to retrieve data. Even improvement in reach measurement and less operating time can be achieved with ANN. This is an improvement in performance if compared to conventional distance relays. Also, the use of ANNs can make it possible to extend the first zone reach of the relays, enhancing system security [1].

In this paper, back propagation feed forward neural network is considered. All types of faults are discussed i.e. single phase to ground faults, double phase faults, double phase to ground faults and three phase fault. Results obtained shows that the proposed scheme is able to distinguish the condition whether there is a fault or not, type of fault and also locates the zone in which fault is present in order to isolate the fault. The network is divided into three different zones i.e. $\mathrm{Z} 1, \mathrm{Z} 2$ and $\mathrm{Z} 3$. Simulation is done using sim power system and neural network in MATLAB. 


\section{BACKPROPAGATION NEURAL NETWORK}

In multi-layer feed forward networks, the processing elements are arranged in layers and only the elements in adjacent layers are connected. It has minimum of three layers of elements i.e.
(i) Input layer
(ii) Hidden layer
(iii) Output layer

It has been proved from experiments that number of hidden neurons should be atleast greater than the sum of squares of neurons in input layer and those in output layer. The information propagation is only in forward direction, there are no feedback loops but error is propagated back in the other direction also to change the weights to obtain a better performance [2].

Training of multi-layer feed forward networks by back propagation algorithm:-

Multi-layer network involves the minimization of least mean squared error. The BPA also known as generalized delta rule and is based upon the concept of property of convergence.

In this algorithm, initially input vector $\mathrm{I}_{\mathrm{i}}$ is set and weight $\mathrm{W}_{\mathrm{ij}}$ is assumed and then potential $U_{j}$ is calculated. The output $\mathrm{H}_{\mathrm{j}}$ is obtained by non-linear transfer function from potential $\mathrm{Uj}$ and $\mathrm{V}_{\mathrm{kj}}$ is assumed. Similarly, internal potential $\mathrm{S}_{\mathrm{k}}$ of the $\mathrm{k}$ th unit of output layer is calculated and then output $\mathrm{O}_{\mathrm{k}}$ is calculated by non-linear transfer function of the internal potential $\mathrm{S}_{\mathrm{k}}$. Error emission is calculated by taking difference between target value $\mathrm{T}_{\mathrm{k}}$ and the output value $\mathrm{O}_{\mathrm{k}}$. Modification of weights is done at two levels as shown in fig1 to obtain better performance [3].

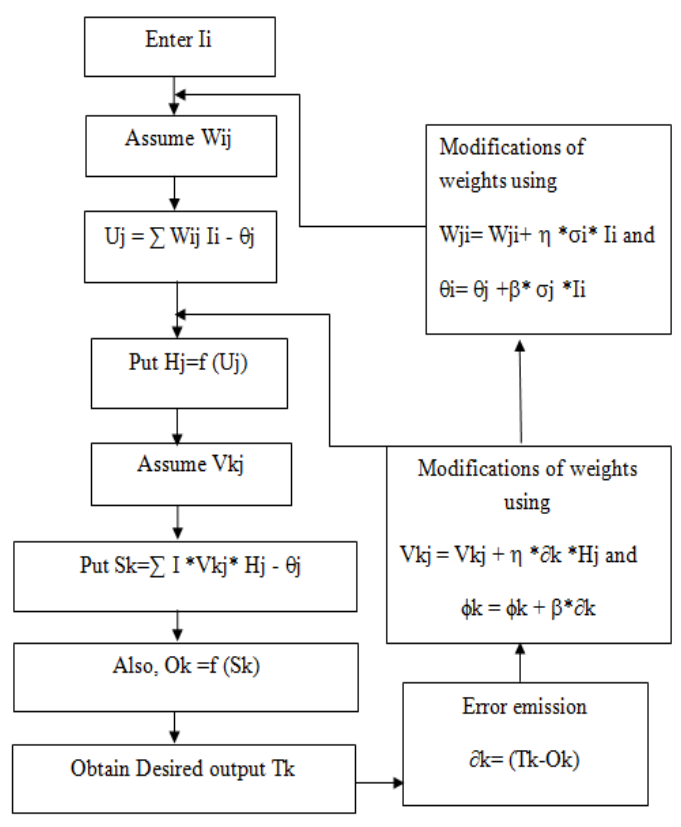

Fig 1:- Back propagation algorithm
The main attraction of back propagation algorithm is that it is applicable to wide variety of networks and this algorithm has become the focus point of researches on neural computing. There is only one drawback that training time required is long.

\section{TRANSMISSION LINE MODEL}

Consider power system model which is simulated in MATLAB is shown in fig 2 and 3 in which no fault and various types of faults conditions are created [4]. The difference between two buses i.e. bus 1 and bus 2 is $300 \mathrm{~km}$. Distributed line L1 and L2 is considered and the distance of line L1 is considered as d1 then the remaining distance will be 300-d1. Current and voltage values are taken from bus1 and saved in workspace from where it is provided as an input to ANN [5].

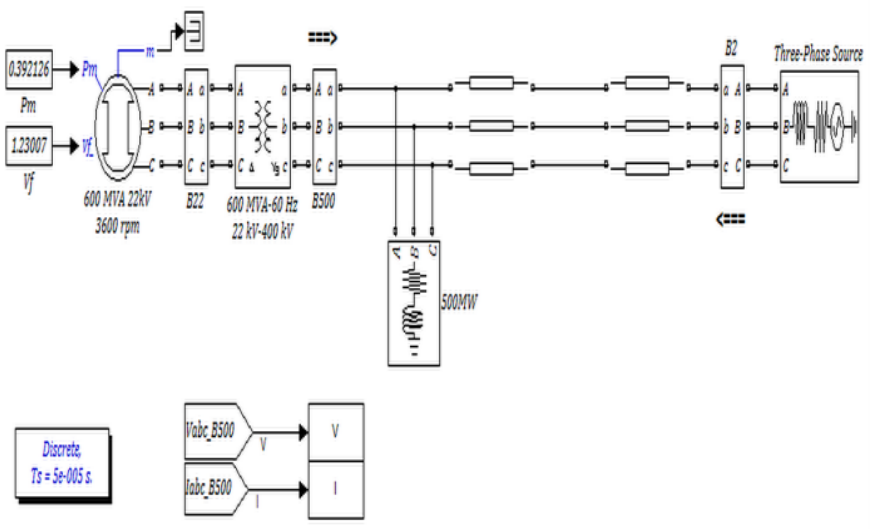

Fig 2:- Simulated model without fault.

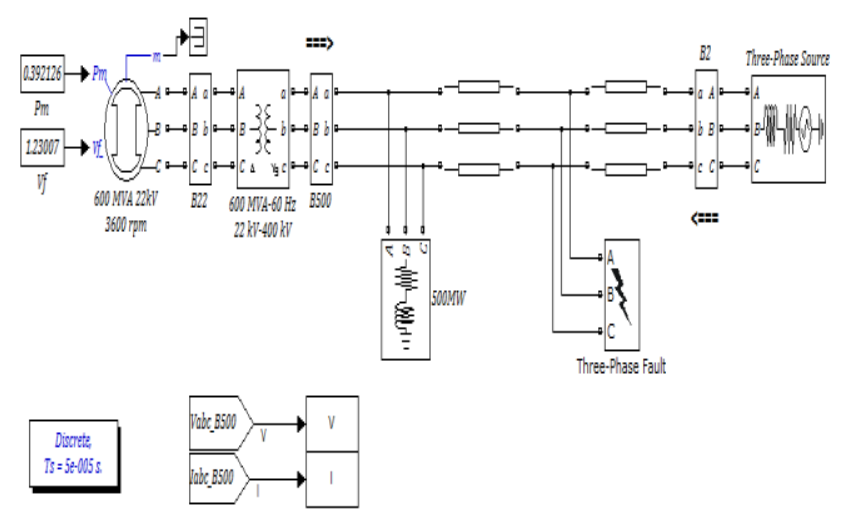

Fig 3:-Simulated model with various types of faults.

\section{DESIGN OF ARTIFICIAL NEURAL NETWORK}

The design of ANN network is done using two networks in which one is for fault detection as well as classification while other is for fault location for isolation. 


\subsection{Fault Detection and Classification}

Input data is taken from power system simulated model having condition of no-fault plus different types of faults. Then, training is provided to the data which is obtained from generated training sequence, testing of the trained sequence is done and finally the condition of fault or no fault is checked ([6],[7]). Various types of faults i.e. single line to ground fault, line to line fault and double line to ground fault are classified [8]. Number of neurons considered for hidden layer is 25.

\subsection{Fault Isolation/Location}

Further, after detecting the fault, the next task is to locate the fault for isolation. The network is expected to identify the location of fault by classifying the identified fault into three different zones, namely Z1, Z2 and Z3. The distance covered by zone $\mathrm{Z} 1$ is $0-100 \mathrm{~km}, \mathrm{Z} 2$ is $100-200 \mathrm{~km}$ and $\mathrm{Z3}$ is $200-300 \mathrm{~km}$ [9]. Five hidden layers are used with 6, 5, 4, 3 and 2 neurons respectively.

\subsection{Training and Testing}

Firstly, system is simulated for no fault condition and minimum and maximum values of three phase voltage and current are saved. Then, system is again simulated for various faults one by one at different locations i.e. 30,60,90,120,150, $180,210,240,270 \mathrm{~km}$ from busbar and the minimum and maximum values of three phase currents and voltages are saved. Two objects for feed forward back propagation neural network for fault detection/classification and location are created. Then the two networks are trained with input as maximum and minimum values of voltages and currents and corresponding output as fault type and fault zone.

Testing is done and it will provide type of fault and location of fault for isolation [10].

\subsection{Results obtained from Training and Testing Data}

The process involving training and testing of different network configurations can be carried out until satisfactory results are achieved [1]. Following are the results obtained from training and testing the data in neural network. Fig 4 and 5 are the results obtained from detection of fault while fig 6 and 7 are the results after locating the fault.

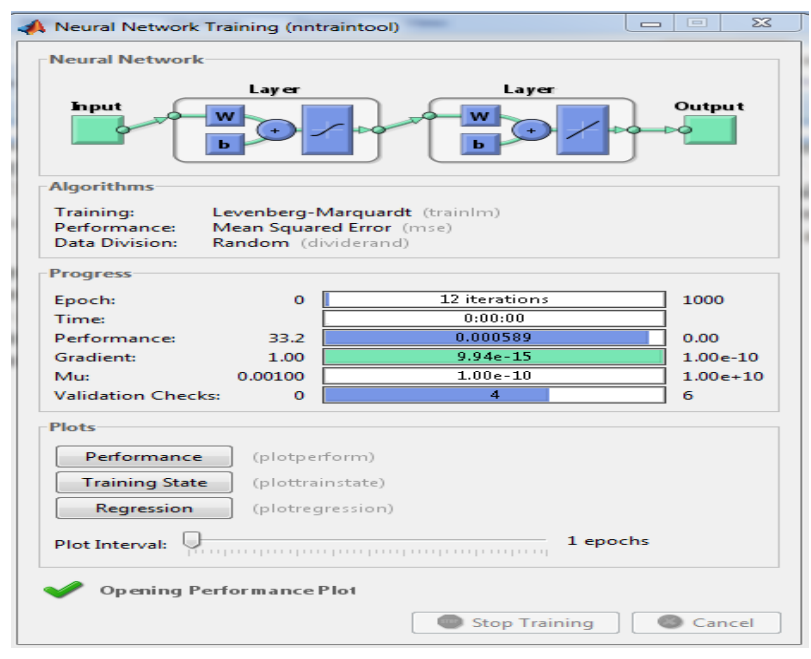

Fig 4:- Training and testing data results for fault location

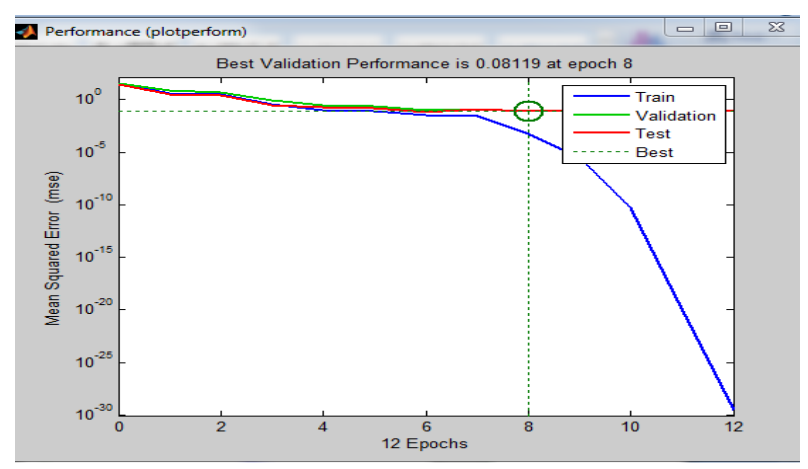

Fig 5:- Performance plot for fault location

In first network, the numbers of iterations used for the training process are 12. Best validation performance is achieved at 0.08119 at epoch 8 .

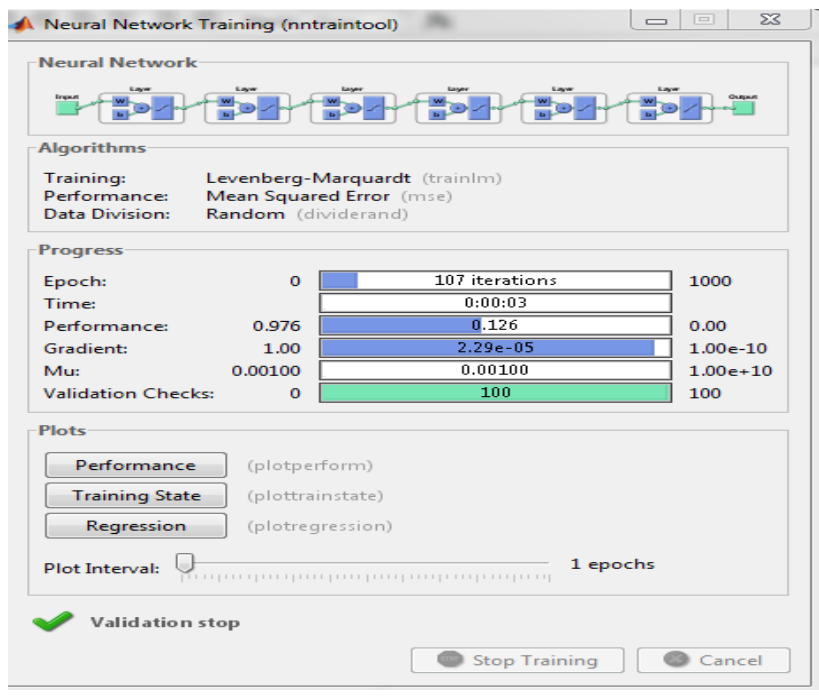

Fig 6:- Training and testing data results for fault location 


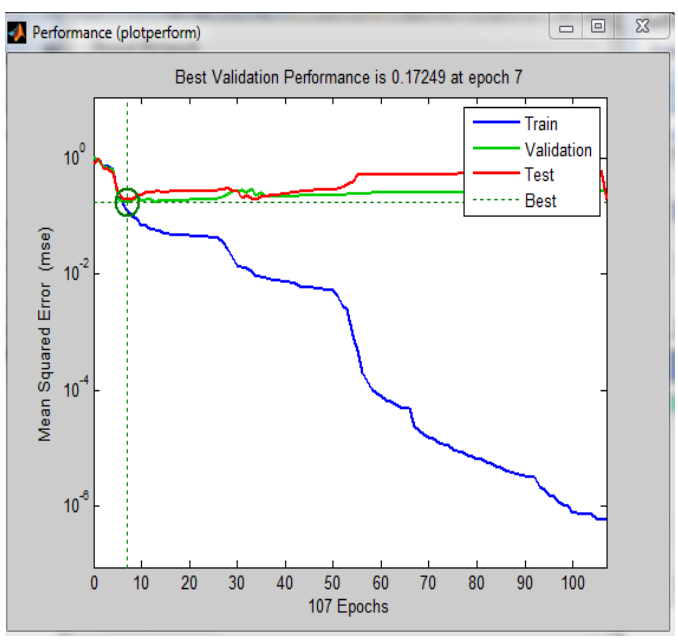

Fig 7:- Performance plot for fault location

The results obtained from fault location network are that the numbers of iterations used for the training process are 107. Best validation performance is achieved at 0.17249 at epoch 7 .

The performance plot shows the value of the mean squared error versus iteration. It plots training, validation, and test performances. The validation and test curves are very similar. If the test curve had increased significantly before the validation curve increased, then it is possible that some over-fitting might have occurred.

\section{RESULTS}

Thus, after obtaining the trained data from neural network it is obtained that whether there is a fault or not and if it is detected then it is identified which type of fault is there. After this, fault distance is calculated which indicates that the fault is present in which zone for isolation.

\section{CONCLUSIONS}

This paper presents the distance protection scheme for transmission line using back-propagation feed forward neural network. This method is less time consuming, accurate, no need for communication link to retrieve data, adapt according to changing conditions due to fault and also reach measurement can be improved. Conclusion is drawn from the results obtained that this method of fault detection, classification and isolation/location is considered to be the accurate one.

\section{FUTURE SCOPE OF THIS WORK}

In this work, minimization of the mean squared error is achieved using Back-propagation feed forward neural network but further this error can be much more minimized by using ANIF to further improve the performance of the relay and this can be considered as a potential area for future study.
Also, this work can be extended for applying the similar technique to the transmission line having FACTS devices. Fault detection and isolation task may get considerably affected by various conditions (such as fault -resistance, inception angle, location) in the presence of FACTS devices and still a critical task to be studied further.

\section{REFERENCES}

[1] Mr.Abhijit A. Dutt, Mr. Atul N. Kadua, "Pattern Recognition Method For Detecting Fault in EHV Transmission Lines", 2010 International Conference on Mechanical and Electrical Technology (ICMET 2010). M. Ananda Rao and J. Srinivas, "Neural Networks Algorithms an Applications", Narosa publication, 2005 .

D.V. Coury and D.C. Jorge, "The Back propagation Algorithm Applied to Protective Relaying", IEEE, 1998.

Isha Awasthi and Ritu Agarwal, "Implementation of Simulink in Protection of Transmission Lines", International Journal of Advances in Electrical and Electronics Engineering.

[5] M Oleskovicz, D V Coury and R K Aggarwal, "A Complete Scheme for Fault detection, classification and location in Transmission lines using Neural Networks", Development in power system protection, Conference Publication, IEEE, 2001.

[6] Rajveer Singh, "Fault Detection of Electric Power Transmission Line by Using Neural Network", International Journal of Emerging Technology and Advanced Engineering Volume 2, Issue 12, December 2012.

[7] Anamika Yadav, A.S. Thoke, "Transmission line fault distance and direction estimation using artificial neural network", International Journal of Engineering, Science and Technology Vol. 3, No. 8, 2011, pp.110-121.

[8] LL Lai, "Application of Neural Networks to Fault Classification and Protection", proceedings of the $4^{\text {th }}$ International Conference on Advance in Power System Control, Operation and Management, HongKong, November,1997.

[9] Tahar Bouthiba "Fault Location in EHV Transmission Lines using Artificial Neural Networks", Int. J. Appl. Math. Comput. Sci., 2004, Vol. 14, No. 1, 69-78.

[10] Eisa Bashier and M. Tayeb omer A/aziz A/Rhim, "Transmission Line Faults Detection, classification and location using Artificial Neural Network", IEEE, 2012. 